medgen $2019 \cdot 31: 372-382$

https://doi.org/10.1007/s11825-020-00287-3

Online publiziert: 12. Februar 2020

(c) Der/die Autor(en) 2020

\author{
Mirko Rehberg' · Julia Etich ${ }^{2}$ Lennart Leßmeier ${ }^{3} \cdot$ Helge Sill $^{1} \cdot$ Christian Netzer $^{3}$. \\ Oliver Semler ${ }^{1}$ \\ 'Medizinische Fakultät und Uniklinik Köln, Klinik und Poliklinik für Kinder- und Jugendmedizin, \\ Universität zu Köln, Köln, Deutschland \\ ${ }^{2}$ Dr. Rolf M. Schwiete Forschungsbereich für Arthrose, Orthopädische Universitätsklinik Friedrichsheim \\ gGmbH, Frankfurt/Main, Deutschland \\ ${ }^{3}$ Medizinische Fakultät und Uniklinik Köln, Institut für Humangenetik, Universität zu Köln, Köln, \\ Deutschland
}

\title{
Osteogenesis imperfecta - Pathophysiologie und aktuelle Behandlungsstrategien
}

gesichert werden. Leichtere Verlaufsformen führen erst in kritischen Lebensabschnitten (Aufrichtung, erste Schritte) zu Problemen und werden dann diagnostiziert, wobei immer die Differentialdiagnose der Kindesmisshandlung beachtet werden sollte. Auch in der Phase des raschen Körperwachstums (pubertärer Wachstumsspurt) kommt es nochmals zu einer Erhöhung des Frakturrisikos. Sobald die Körperlängenentwicklung abgeschlossen ist, nimmt die Frakturrate im Erwachsenenalter deutlich ab [2].

Zur Abklärung von Frakturen steht die Röntgenuntersuchung an vorderster Stelle. Die langen Röhrenknochen können Deformierungen aufweisen, die Kortikalis ist meist dünn und der gesamte Knochen erscheint auf der konventionellen Röntgenaufnahme osteopen. Die Röntgenaufnahme einer seitlichen Wirbelsäule (Lenden- und Brustwirbelsäule) ist unverzichtbar, um nach Kompressionsfrakturen oder Deformierungen (Fisch- oder Keilwirbelkörper) zu suchen, die ein starkes Indiz für eine reduzierte Knochenstabilität sind (s. - Abb. 1a). Die metaphysären Bereiche können aufgetrieben sein und durch versprengtes Knorpelgewebe kann es zu den sog. „Popcorn-Metaphysen“ kommen (s. A Abb. 1b).

Bei der Untersuchung muss nicht nur der skelettale Befund erhoben werden, sondern es müssen auch die nicht ossären Symptome beachtet werden [2]. Hierzu gehört im Rahmen der Kollagen- synthesestörung die Hypermobilität der Gelenke, die Muskelhypotonie - häufig mit einem Knick-Senkfuß verbunden der Kleinwuchs, die Blauverfärbung der Sklera bei ca. $50 \%$ der Betroffenen und die Beteiligung der Zähne im Rahmen einer Dentinogenesis imperfecta Typ 1 [3]. Die Laboruntersuchungen sind bei OI meist wenig spezifisch und spiegeln eine „High-turnover“-Osteoporose im Sinne eines gestörten Verhältnisses von Auf- und Abbau wider. Dabei versucht der Organismus das fehlerhafte Kollagen schnell $\mathrm{zu}$ beseitigen und durch viel neues, zum Teil histologisch übermineralisiertes Kollagen $\mathrm{zu}$ ersetzen. Laborchemisch zeigt sich der Prozess in einer erhöhten alkalischen Phosphatase (AP) sowie erhöhten Osteoblastenund Osteoklastenmarkern (Osteoblastenmarker bzw. Marker des Knochenaufbaus: Osteocalcin; Osteoklastenmarker bzw. Marker des Knochenabbaus: Desoxypyridinolin, $\beta$-CrossLaps) bei unauffälligen Werten für die Mineralisierung des Knochens. Sollte sich aus diesen Untersuchungen keine Diagnose stellen lassen, sollte eine Knochendichtemessung erfolgen. Wenn die „Dual Energy X-ray Absorptionmetry“ (DXA) verwendet wird, muss im Kindes- und Jugendalter auf die Verwendung von Referenzwerten geachtet werden, die auf Alter und Körperlänge bezogen sind, da sonst kleinwüchsige Kinder immer eine erniedrigte Knochenflächendichte im Vergleich zu gleichaltrigen Kin- 


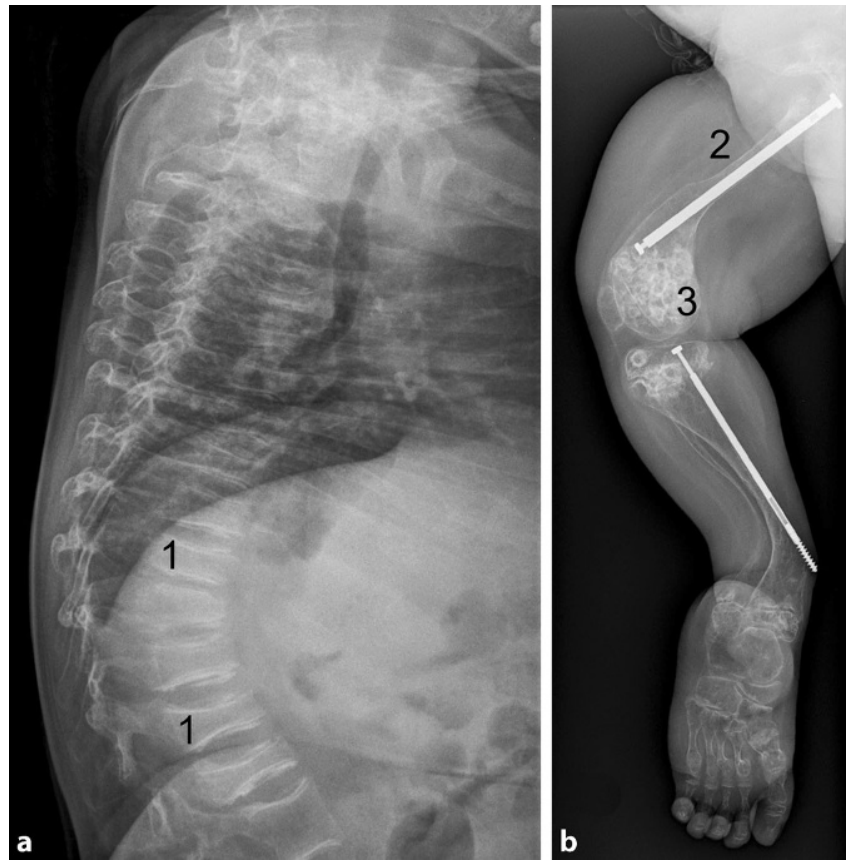

Abb. $1 \Delta$ Röntgenaufnahme der lateralen Wirbelsäule (a) einer Patientin mit einer schweren Verlaufsform einer Osteogenesis imperfecta, verursacht durch eine qualitative Störung in $\mathrm{COL} 1 \mathrm{A2}$. Es sind ausgeprägte Wirbelkörperveränderungen (z. B. 1, unauffälliger Wirbelkörper hat 2:3- bis 4:5-Verhältnis von Höhe zu Breite) zu sehen. Aufnahme eines Beines der gleichen Patientin mit Z. n. operativer Frakturversorgung und Begradigung mit einem Teleskopnagel (2) mit typischer dünner Kortikalis und Auftreibung der Epiphysen mit eingesprengten Knorpelfragmenten, die zum Bild der Popcorn-Epiphysen (3) führt. Die Verankerung des Nagels in der distalen Unterschenkelepiphyse scheint in der vorliegende Ebene nicht mehr gegeben und somit nicht optimal (b).

dern haben. Bei etwas älteren Kindern und Erwachsenen sollte eine „periphere quantitative Computertomographie“ (pQCT) erfolgen. Mit dieser Methode kann die physikalische Dichte des Knochens unabhängig von der Größe des Patienten bestimmt werden und es ist eine detaillierte Analyse der kortikalen und spongiösen Strukturen möglich [4].

Grundsätzlich empfiehlt sich, eine molekulargenetische Sicherung der Diagnose anzustreben. Da es bisher keine individuelle Genotyp-Phänotyp-Korrelation bei OI gibt, ermöglicht die genetische Analyse keine konkrete Vorhersage des Krankheitsverlaufs, aber die Kenntnis der Mutation verbessert die Aufklärung der Familien in Bezug auf das Wiederholungsrisiko bzw. die Weitervererbung [2]. Zusätzlich kann die Diagnose zukünftig ein spezifisches Monitoring oder eine spezifischere Therapie ermöglichen (siehe therapeutische Perspektiven).

Bei leichten Verlaufsformen sollte eine genetische Untersuchung hingegen aus sozialmedizinischer Sicht kritisch disku- tiert werden. Die genetische Diagnose kann bei geringer klinischer oder therapeutischer Relevanz negative Effekte auf die Entwicklung des Kindes haben, da es z.B. zu einer Überprotektion oder Problemen bei der sozialen Integration (z.B. Sportverein) kommen kann.

\section{Konventionelle medikamentöse Therapie}

Die medikamentöse Therapie ist derzeit rein symptomatisch. Für alle Schweregrade gilt, dass die Sicherstellung einer ausreichenden Mineralisierung des Knochens notwendig ist. Da es sich bei der OI nicht um eine Mineralisierungsstörung handelt, sondern in den meisten Fällen um eine Synthesestörung, ist eine über die normale Versorgung hinausgehende Substitution mit Calcium und Vitamin D nicht sinnvoll [1]. Bei moderat oder schwer betroffenen Patienten, die mehr als 2 Frakturen pro Jahr erleiden, oder die von Wirbelkörperfrakturen und chronischen Knochenschmerzen betroffen sind, hat sich besonders im Kindes- und Jugendalter eine Therapie mit intravenösen Bisphosphonaten bewährt. Diese antiresorptiven Medikamente binden sich an die Hydroxylapatitkristalle am Knochen und führen bei Resorption durch Osteoklasten im Rahmen des Remodelling zu deren Apoptose [5]. Durch Bisphosphonate kann die Resorption reduziert werden und dadurch kommt es bei weiter stattfindendem Aufbau zu einer Zunahme an Knochenmasse, wenngleich Bisphosphonate insgesamt eine Reduktion des gesamten Knochenumsatzes bewirken können. In Metanalysen konnte kein signifikanter Effekt auf die Frakturrate nachgewiesen werden [6]. Dennoch erscheint die NutzenRisiko-Abwägung für eine Bisphosphonattherapie zu sprechen. Durch den nachgewiesenen Zugewinn an Mobilität und die reduzierten Schmerzen haben die behandelten Kinder eine größere Teilhabe an Alltagsaktivitäten [7]. Dies kann für sich schon mit einem höheren Verletzungsrisiko einhergehen. Dadurch hat das Medikament zwar keinen direkten Effekt auf die Frakturrate, dafür auf Teilhabe und Lebensqualität. Zudem ist im Kindesalter ein Wiederaufbau der Wirbelkörper gut belegt [7], als Hinweis für die knochenstabilisierende Wirkung. Bei der ersten Gabe von intravenösen Bisphosphonaten kommt es häufig zu einer "Akutphase-Reaktion“ mit Fieber und grippeähnlichen Symptomen, die bei weiteren Gaben nicht mehr auftreten. Langfristige Nebenwirkungen sind bisher nicht beschrieben, aber die Erfahrungen mit dieser Wirkstoffgruppe sind begrenzt. Bisher wurde weder das vermehrte Auftreten von atypischen Femurfrakturen noch von Kiefernekrosen bei Kindern und Jugendlichen beschrieben [8]. Der Einsatz von Bisphosphonaten ist bei Kindern und Jugendlichen mit einer schweren Verlaufsform einer OI weitgehend als Standard etabliert, wenngleich es in den meisten Ländern keine für OI zugelassenen Präparate gibt [2]. Im Erwachsenenalter erfüllen die Patienten meist die Kriterien der Osteoporose und werden dementsprechend behandelt. Im Kindesalter ist jeder Einsatz von Bisphosphonaten ein ,individueller Heilversuch“, über den aufgeklärt werden muss. 
medgen 2019.31:372-382 https://doi.org/10.1007/s11825-020-00287-3

(c) Der/die Autor(en) 2020

M. Rehberg · J. Etich · L. Leßmeier · H. Sill · C. Netzer · O. Semler

Osteogenesis imperfecta - Pathophysiologie und aktuelle Behandlungsstrategien

\section{Zusammenfassung}

Die Osteogenesis imperfecta (OI) ist eine angeborene Erkrankung des Knochens und Bindegewebes. Sie geht mit einer erhöhten Frakturneigung, Deformierung der Extremität, aber auch mit extraskelettalen Symptomen einher. Nach einer kurzen Darstellung von Klinik, Diagnostik und aktueller Therapie folgt ein umfassender Überblick über die genetischen und pathophysiologischen Grundlagen der Erkrankung und die daraus abgeleiteten zukünftigen therapeutischen Möglichkeiten.

Ungefähr $80 \%$ der Patienten haben eine Mutation in den Kollagen-Genen COL1A1 und COL1A2. Bei diesen Patienten ist für das Kollektiv keine klare Genotyp-PhänotypKorrelation beschrieben. Stoppmutationen führen in der Regel zu einem quantitativen
Kollagendefekt, wodurch weniger normales Kollagen gebildet wird und ein eher leichter Phänotyp entsteht. Missense-Mutationen führen zu strukturell verändertem Kollagen (qualitativer Defekt) und zu einem schwereren Phänotyp. Trotzdem gibt es Unterschiede und Vorhersagen über den individuellen Verlauf sind nur sehr eingeschränkt möglich. Neben Veränderungen in den Kollagen-Genen gibt es Mutationen, welche die Kollagenmodifikation und die Kollagensekretion betreffen. Eine eigene Gruppe bilden Gene, welche an der Osteoblastendifferenzierung beteiligt sind. Wie auch bei den weiteren, nicht näher zugeordneten Genen sind dies häufig übergeordnete Gene, deren Funktion in der Osteogenese nicht völlig verstanden ist.
Abgeleitet aus den pathophysiologischen Grundlagen, können vorhandene Medikamente zukünftig womöglich zielgerichtet eingesetzt werden. So ist der "ReceptorActivator-of-Nuclear-Factor-Kappa BLigand" (RANKL)-Antikörper Denosumab spezifischer als Bisphosphonate und wird schon heute bei OI-Typ VI (SERPINF1) verwendet. Weitere Medikamente wie AntiSklerostin oder Stammzelltherapien werden unter Berücksichtigung der Pathophysiologie aktuell entwickelt.

Schlüsselwörter

Kollagen · Therapie · Mutation · Bisphosphonate $\cdot$ Denosumab

\section{Osteogenesis imperfecta: Pathophysiology and current treatment strategies}

\section{Abstract}

Osteogenesis imperfecta $(\mathrm{OI})$ is a hereditary disease of the bones and fascia. It is associated with an increased tendency to fracture, deformities of the extremities, and extraskeletal signs. A short description of the clinical course, diagnostic recommendations and the current treatment are followed by an extensive overview of the genetic and pathophysiological background of the disease and future therapeutic options. Approximately $80 \%$ of patients present with mutations in genes coding for collagen (COL1A1/A2). In these patients, no clear correlation between phenotype and genotype is described for the collective. Stop mutations usually cause a quantitative collagen defect, which results in less normal collagen and a mild phenotype. Missense mutations lead to structurally changed collagen (qualitative defect) and to a more severe phenotype. Nonetheless, there is high variability and it is difficult to predict the course of an individual patient. In addition to changes in the collagen coding genes, there are mutations that affect the modification and secretion of collagen. A specific group consists of genes involved in the differentiation of osteoblasts. As with the other genes (which are not referred to in more detail), these are often superior genes, whose function in osteogenesis is not fully understood.
Based on the pathophysiological principles, existing treatments may well be more precisely deployed in the future. An example is the receptor activator of nuclear factor kappa-B ligand (RANKL) antibody denosumab, which is more specific than bisphosphonates, and is already used in OI type VI (SERPINF1). Further treatments such as antisclerostin or stem cell therapies are currently being investigated with a focus on pathophysiology.

Keywords Collagen · Therapy · Mutation · Bisphosphonates $\cdot$ Denosumab

\section{Chirurgisch-orthopädische Therapie}

Die chirurgische Behandlung untergliedert sich in die akute Frakturversorgung und in die Korrektur von Deformierungen. Akute Frakturen sind bei Patienten mit OI nur selten disloziert und können meist konservativ durch Immobilisation behandelt werden. Dies ist meist auch die einzige Option in den ersten 2 Lebensjahren, da der Knochen in diesem Alter für operative Maßnahmen zu klein und zu dünn ist. Bei älteren Kindern oder bei Vorliegen ausgeprägter Deformierungen erfolgt eine operative Behandlung. Im Kindes- und Jugendalter, also wenn das Skelettsystem noch wächst, sollten Teleskopnägel in den Knochen eingebracht werden, die jeweils jenseits der Wachstumsfuge verankert werden und sich während des Wachstums in die Länge ziehen. Dadurch erhält der Knochen von innen eine Schienung, die bei weiteren Frakturen vor einer Dislokation schützt. Der Gebrauch von Platten und einem Fixateur externe sollte vermieden werden, da es hierbei an den Eintrittsstellen und am Ende der Platte $\mathrm{zu}$ „Sollbruchstellen“ kommt. Zur Achskorrektur von Kniefehlstellungen kann auch eine Epiphysenklammerung/ Epiphyseodese durchgeführt werden [9].

\section{Physiotherapeutische/ rehabilitative Therapie}

Alle Maßnahmen, die dazu dienen die Knochenstabilität der Patienten zu er- 
höhen als auch die Deformierungen zu korrigieren, müssen als gemeinsames Ziel die Verbesserung der Mobilität und Selbstständigkeit der Patienten haben. $\mathrm{Da}$ die OI auch eine Schwäche des Sehnen- und Bandapparates bedingt und viele Patienten auch eine Muskelhypotonie aufweisen, ist eine regelmäßige Kräftigung der Muskulatur zur Verbesserung der Beweglichkeit entscheidend [10]. Zudem müssen die Betroffenen nach Operationen lernen, welche neuen Bewegungsabläufe nun möglich sind. Insgesamt erreichen die meisten schwerer Betroffenen ihre motorischen Meilensteine später als andere Kinder [11]. Aufgrund der Frakturen haben viele Betroffene Angst, neue Bewegungsmuster zu probieren, was beim Training beachtet werden muss. Ziel der Rehabilitationsmaßnahmen muss immer ein Zugewinn an Lebensqualität und Mobilität sein [1]. Zusätzlich wird durch eine Kräftigung der Muskulatur ein osteoanaboler Stimulus ausgeübt, über den es zu einer Zunahme der Synthese extrazellulärer Matrix kommt. Obwohl die Funktion der Osteoblasten bei den meisten Formen einer OI eingeschränkt ist, so ist die Nutzung der Muskulatur dennoch die beste Möglichkeit den Knochenaufbau anzuregen [12].

Es ist in den vergangenen 2 Jahrzehnten gelungen, die Behandlung von OIPatienten zu verbessern, nichtsdestotrotz ist die derzeitig angewendete Behandlung rein symptomatisch und spiegelt das immer komplexere Verständnis über die pathophysiologischen Vorgänge bei OI nicht wider. Diese müssen in Zukunft besser untersucht werden, um daraus individualisierte therapeutische Angebote entwickeln zu können.

\section{Pathophysiologie}

\section{Kollagen-Biosynthese, Modifikation und Sekretion}

\section{Kollagene OI-Typ I-IV}

Heterozygote Mutationen in den Genen COL1A1 und COL1A2 sind die häufigste Ursache für eine OI. Die Größe der Gene erklärt die Vielzahl der bekannten Mutationen und einen Teil der Heterogenität der klinischen Symptomatik. Bisher konnte keine Genotyp-Phänotyp-Korrelation identifiziert werden und es ist keine Zuordnung zu den klinischen Typen aufgrund der Mutation möglich. Dennoch können 2 unterschiedliche Pathophysiologien beschrieben werden: Stopp-Mutationen führen zu einer Haploinsuffizienz. Somit haben die Betroffenen zwar eine reduzierte Menge an Kollagen, dieses ist jedoch von normaler Qualität. Der Phänotyp ist meist weniger stark ausgeprägt. Demgegenüber führen andere Mutationen (meist Glycin-Mutationen, Missense-Mutationen) zu einer qualitativen Störung der extrazellulären $\mathrm{Ma}$ trix, da sich die Kollagenfibrillen nicht regulär bilden können. Dies hat in der Regel eine schwerere klinische Verlaufsform zur Folge [13]. Durch die qualitative Störung und die unzureichende Stabilität der Knochensubstanz wird zusätzlich auch der Knochenabbau angeregt, da der Körper versucht, den qualitativ gestörten Knochen abzubauen. Durch die verminderte Knochenstabilität und den Abbau stimuliert, produzieren die Osteoblasten möglichst viel Knochensubstanz, die jedoch von reduzierter Qualität ist. Dadurch ergibt sich klinisch und laborchemisch das Bild einer „High-turnover“Osteoporose.

In den vergangenen Jahren wurden weitere Gene identifiziert, die zu dem klinischen Krankheitsbild der OI führen. Eine Liste dieser Gene, zusammen mit den dadurch veränderten Proteinen und den Vererbungswegen zeigt • Tab. 1 und die Lokalisation der meisten Störungen innerhalb des Kollagen-Synthesewegs ist in $\bullet$ Abb. 2 dargestellt.

Veränderungen in mehreren Genen können die Symptome einer OI erzeugen, bewirken aber keine Veränderung der Kollagensequenzen, sondern beeinflussen seinen Biosyntheseweg. Dieser Prozess umfasst multiple Schritte und bedarf einer Vielzahl an Proteinen für die posttranslationale Modifikation, Faltung, Transport und Qualitätskontrolle des Kollagens.

\section{P4HB}

Der helikale Bereich des Kollagenmoleküls ist reich an Prolinen, die in repetitiven Sequenzen angeordnet sind. Nach der Translation des Prokollagens ins raue endoplasmatische Retikulum (ER) ist einer der ersten Schritte in der posttranslationalen Modifikation des Kollagens die Hydroxylierung der helixständigen Prolinreste zu Hydroxyprolin. Die Hydroxylierung führt $\mathrm{zu}$ einer größeren Stabilität der Kollagen-Tripelhelix; die meisten Prolyl-4-Hydroxylierungen im Kollagenmolekül werden dabei vom tetrameren Prolyl-4-Hydroxylasekomplex bewerkstelligt. Eine Untereinheit dieses Komplexes wird durch das Gen $P 4 H B$ kodiert. Das $P 4 H B$-kodierte Protein PDI (Protein-Disulfid-Isomerase) kann auch als ein molekulares Chaperon dienen und Disulfidbrückenbildung regulieren. Allerdings ist der Pathomechanismus der identifizierten $\mathrm{P} 4 \mathrm{HB}$-Mutationen bis dato nicht bekannt. Mutationen führen zum Cole-Carpenter-Syndrom Typ 1, werden klinisch in Einzelfällen aber auch als mittelschwere bis schwere OI beschrieben [14].

\section{P3H1, CRTAP, PPIB - OI-Typ VIII, VII, IX}

Die Prolyl-3-Hydroxylierung an wenigen bestimmten Prolinresten im Kollagenmolekül wird durch drei verschiedene Prolyl-3-Hydroxylase-Isoformen durchgeführt. Der Komplex aus P3H1 (Prolyl-3-Hydroxylase 1), CRTAP (cartilage associated protein) und PPIB (Peptidylprolyl-cis-trans-Isomerase B oder Cyclophilin B) ist verantwortlich für die Prolin-986-Hydroxylierung der a-Kette des Kollagens. Rezessive Mutationen in allen drei Genen führen zu unterschiedlich stark ausgeprägter OI. Dabei kommt es zu einer Verminderung der Prolin-986Hydroxylierung und somit zu einer Verzögerung der Kollagenfaltung, einhergehend mit einer übermäßigen Modifikation [15]. Oft kommt es in Patientenzellen zur intrazellulären Retention und Aggregatbildung des übermodifizierten Kollagens, was zum ER-Stress und anschließendem Zelltod führen kann. Im Prozess der Hydroxylierung sorgt Cyclophilin B für die cis-trans-Isomerisierung der Kollagen-Prolyl-Peptidbindung und zusammen mit FKBP65, einem molekularen Chaperon, verhindert es die vorzeitige Assoziation der Prokollagen- $\alpha$-Ketten. Außerdem kann Cyclophilin B mit Lysylhydroxylase 1 (LH1) interagieren sowie 
Tab. 1 Liste der derzeit bekannten Gene, in denen Mutationen eine Ol oder überlappende Erkrankung auslösen können.

\begin{tabular}{|c|c|c|c|c|c|}
\hline Gen & Protein & OMIM-Gen & Erkrankung & Vererbung & OMIM-Erkrankung \\
\hline COL1A1 & Collagen a $1(\mathrm{I})$ chain (COL1A1) & 120150 & Ol-Typ I & $A D$ & 166200 \\
\hline \multirow[t]{3}{*}{ COL1A2 } & \multirow[t]{3}{*}{ Collagen a2(I) chain (COL1A2) } & \multirow{3}{*}{120160} & Ol-Typ II & $A D$ & 166210 \\
\hline & & & Ol-Typ III & $A D$ & 259420 \\
\hline & & & OI-Typ IV & $A D$ & 166220 \\
\hline IFITM5 & Interferon-induced transmembrane protein 5 (IFITM5) & 614757 & Ol-Typ V & $A D$ & 610967 \\
\hline SERPINF1 & Pigment epithelium-derived factor (PEDF) & 172860 & Ol-Typ VI & AR & 613982 \\
\hline CRTAP & Cartilage-associated protein (CRTAP) & 605497 & OI-Typ VII & AR & 610682 \\
\hline $\begin{array}{l}\text { P3H1 } \\
\text { (früher: } \\
\text { LEPRE1) }\end{array}$ & Prolyl 3 hydroxylase 1 ( $\mathrm{P} 3 \mathrm{H} 1)$ & 610339 & OI-Typ VIII & AR & 610915 \\
\hline PPIB & Peptidyl-prolyl cis-trans isomerase B (PPIB) & 123841 & OI-Typ IX & AR & 259440 \\
\hline SERPINH1 & Serpin $\mathrm{H} 1$ & 600943 & Ol-Typ X & AR & 613848 \\
\hline FKBP10 & $\begin{array}{l}\text { Peptidyl-prolyl cis-trans isomerase FKBP10 (PPlase } \\
\text { FKBP10) } \\
\text { Oder: FK506-binding protein } 10\end{array}$ & 607063 & OI-Typ XI & $A R$ & 610968 \\
\hline SP7 & $\begin{array}{l}\text { Transcription factor Sp7 } \\
\text { alternativ: Zinc finger protein osterix }\end{array}$ & 606633 & OI-Typ XII & $\mathrm{AR}$ & 613849 \\
\hline BMP1 & Bone morphogenetic protein 1 (BMP1) & 112264 & OI-Typ XIII & $\mathrm{AR}$ & 614856 \\
\hline TMEM38B & $\begin{array}{l}\text { Trimeric intracellular cation channel type B (TRIC-B) } \\
\text { alternativ: Transmembrane protein 38B (TMEM38B) }\end{array}$ & 611236 & OI-Typ XIV & AR & 615066 \\
\hline WNT1 & Proto-oncogene Wnt-1 (WNT1) & 164820 & OI-Typ XV & AR & 615220 \\
\hline CREB3L1 & $\begin{array}{l}\text { Cyclic AMP-responsive element-binding protein 3-like } \\
\text { protein } 1 \text { (CR3L1) }\end{array}$ & 616215 & OI-Typ XVI & $\mathrm{AR}$ & 616229 \\
\hline SPARC & Secreted protein acidic and rich in cysteine (SPARC) & 182120 & OI-Typ XVII & AR & 616507 \\
\hline TENT5A & Terminal nucleotidyltransferase 5A (TENT5A) & 611357 & OI-Typ XVIII & $\mathrm{AR}$ & 617952 \\
\hline MBTPS2 & $\begin{array}{l}\text { Membrane-bound transcription factor site-2 protease } \\
\text { (MBTPS2) }\end{array}$ & 300294 & OI-Typ XIX & $\mathrm{XLR}$ & 301014 \\
\hline MESD & LRP chaperone MESD & 607783 & OI Typ XX & AR & 618644 \\
\hline \multicolumn{6}{|c|}{ Andere Formen } \\
\hline P4HB & $\begin{array}{l}\text { Protein disulfide-isomerase (PDI) } \\
\text { alternativ: Prolyl 4-hydroxylase subunit beta (P4HB) }\end{array}$ & 176790 & $\begin{array}{l}\text { Cole-Carpenter-Syndrom } \\
\text { Typ } 1\end{array}$ & $A D$ & 112240 \\
\hline PLOD2 & $\begin{array}{l}\text { Procollagen-lysine,2-oxoglutarate 5-dioxygenase } 2 \\
\text { (PLOD2) }\end{array}$ & 601865 & Bruck-Syndrom Typ 2 & $A R$ & 609220 \\
\hline SEC24D & Protein transport protein Sec24D (SEC24D) & 607186 & $\begin{array}{l}\text { Cole-Carpenter-Syndrom } \\
\text { Typ } 2\end{array}$ & $\mathrm{AR}$ & 616294 \\
\hline PLS3 & Plastin-3 (PLS3) & 300131 & Osteoporose & XLD & 300910 \\
\hline
\end{tabular}

die Kollagenmodifikation und intramolekulare Verlinkung beeinflussen.

Mutationen in CRTAP lösen dabei eher schwere Verlaufsformen aus. Die meisten Kinder haben prä- oder unmittelbar postnatal Frakturen der langen Röhrenknochen. Ein Großteil der bisher publizierten Patienten benötigt als Erwachsene zumindest zeitweise einen Rollstuhl oder andere Hilfsmittel. Mutationen in $P 3 H 1$ werden häufig als ähnlich schwer betroffen beschrieben. Es gibt aber auch Patienten, die mittelschwer betroffen sind, ohne pränatale Frakturen. Diese Patienten können zumeist laufen. Patienten mit PPIB-Mutationen sind häufig schwer, selten mittelschwer betroffen [16]. Die Anzahl der publizierten Fälle ist aber noch sehr gering.

\section{PLOD2}

PLOD2 kodiert das Protein Lysylhydroxylase 2 (LH2), das ähnlich wie die Lysylhydroxylase 1 (LH1) Lysinreste im Kollagenmolekül hydroxyliert. Die Hydroxylierung von Proteinen ermöglicht die kovalente Verlinkung innerhalb des Moleküls und vermittelt somit Zugfestigkeit. Hydroxylysine können anschließend glykosyliert werden. Obwohl die genaue Funktion der Kollagenglykosylierung noch nicht vollständig verstanden ist, ist sie für die Stabilität des Kollagens essenziell. Mutationen im PLOD2-Gen verursachen das Bruck-Syndrom Typ 2, das mittlere bis schwere Skelettveränderungen und progressive Gelenkkontrakturen mit OI-Typ XI teilt [15]. In Bezug auf die Knochenbrüchigkeit liegt eher eine mittelschwere OI vor, für die Lebensqualität entscheidend sind aber eher die progressiven Kontrakturen. Kürzlich wurde gezeigt, dass die Aktivität von LH2 durch die molekularen Chaperone HSP47 und FKBP65 im ER reguliert wird [17]. 


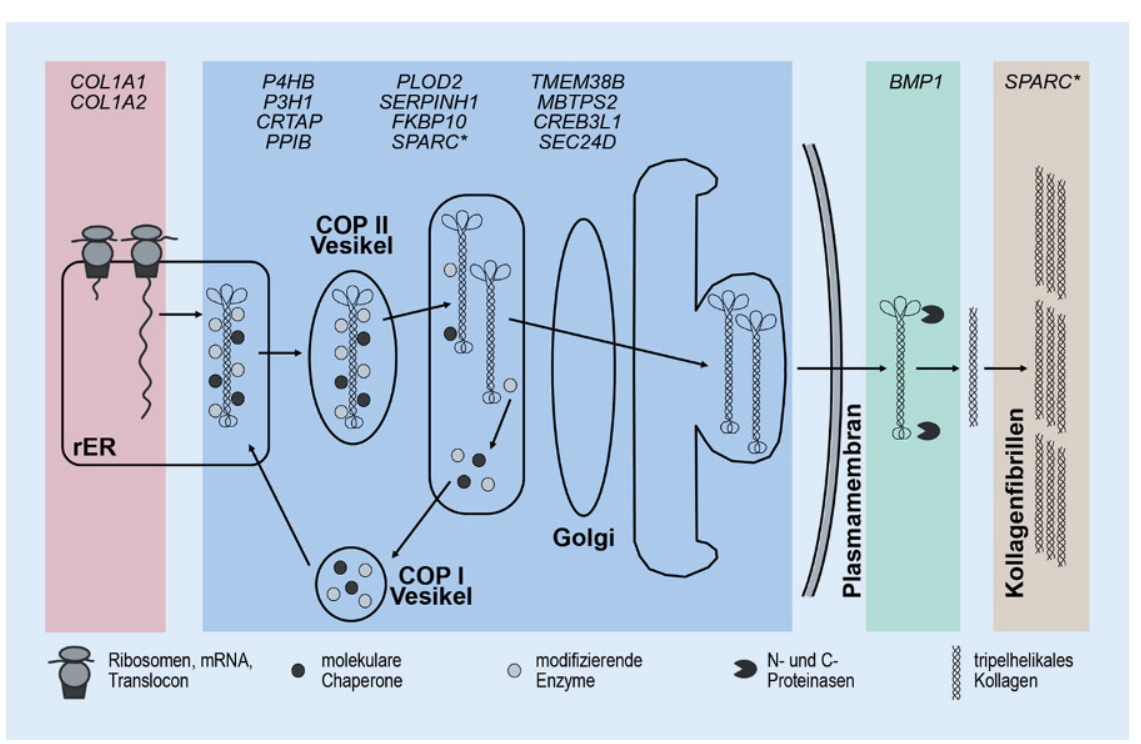

Abb. 2 ム Physiologische Biosynthese des Kollagens und beteiligte Osteogenesis-imperfecta-Gene. Die a1- und a2-Ketten des Kollagen Typ 1 werden in das raue endoplasmatische Retikulum (rER) translatiert. Molekulare Chaperone binden an die sich faltenden Ketten und ermöglichen die Hydroxylierung von Prolin- und Lysinresten durch Hydroxylasen und die anschließende Glykosylierung der Prokollagenketten vor der Tripelhelixbildung. Das Prokollagenmolekül wird im Coat protein complex II (COP) vermittelten vesikulären Transport über das Golgi-Netzwerk in den Extrazellularraum sekretiert. Aufgrund des säuerlichen pH-Wertes im Golgi-Apparat lösen sich Chaperone und modifizierende Enzyme vom Prokollagenmolekül und rezirkulieren COP I-vermittelt zurück zum ER. Im Extrazellularraum werden die $\mathrm{C}$-und $\mathrm{N}$-terminalen Propeptide durch verschiedene Proteinasen abgespalten. Die prozessierten Kollagenmoleküle lagern sich aneinander zu Kollagenfibrillen und werden mineralisiert. An der Biosynthese des Kollagens beteiligte Gene, in denen Mutationen bekanntermaßen eine Osteogenesis imperfecta auslösen können, sind aufgeführt und können der Translation (rot), posttranslationalen Modifikation (blau), Prokollagenprozessierung (grün) oder Fibrillenbildung (orange) zugeordnet werden.SPARC* hat sowohl eine intrazelluläre Funktion als molekulares Chaperon als auch eine extrazelluläre Funktion bei der Mineralisierung der Kollagenmatrix.

\section{SERPINH1 - OI-Typ X}

Molekulare Chaperone zählen zur Familie der Hitzeschockproteine, die die Aggregation von Proteinfaltungsintermediaten verhindern, aber auch bei der Assoziation von Kollagenketten zu übergeordneten fibrillären Strukturen beteiligt sind. Das Chaperon HSP47 (heat shock protein 47) wird vom Gen SERPINH1 kodiert und Mutationen in diesem Gen führen zu einer Instabilität des HSP7-Proteins. Dadurch kommt es $\mathrm{zu}$ einer verzögerten Kollagensekretion sowie veränderten Kollagenstruktur bzw. zur partiellen Retention des Kollagens innerhalb der Zelle [1]. Die wenigen bisher veröffentlichten Mutationen in SERPINH1 verursachen mittelschwere und schwere Verlaufsformen, zum Teil mit starken Deformierungen und pränatalen Frakturen, zum Teil aber auch erst mit Frakturen in den ersten Lebensmonaten[18, 19]. monovalenten Kationenkanal (TRIC-B, trimeric intracellular cation channel type B) kodiert. Dieser Kaliumkanal, welcher unter anderem in der Membran des ER exprimiert wird, ist für eine funktionierende Entleerung intrazellulärer Kalziumspeicher notwendig und spielt eine Rolle in der Zelldifferenzierung. Eine gestörte intrazelluläre Kalziumfreisetzung führt zu einer Fehlregulation der Kollagenmodifikation durch verschiedene Enzyme im ER. In der Folge kommt es zu ER-Stress und einer reduzierten Kollagensekretion. Mutationen in diesem Transmembranprotein sind autosomalrezessiv erblich und gehen mit einer moderaten Form der OI ohne blaugefärbte Skleren, Dentinogenesis imperfecta oder Hörschwäche [14] einher.

\section{MBTPS2 - OI-Typ XIX}

MBTPS2 ist ein X-chromosomal gelegenes Gen, welches für eine membranständige, Zink-Metalloprotease (S2P, site-2 Protease) kodiert. Diese ist mit verschiedenen intrazellulären Signalkaskaden verbunden, unter anderem mit der regulierten Intramembranproteolyse (RIP) der Transkriptionsfaktoren CR3L1, ATF6 und SREBP. Vermittelt über eine verminderte Menge von LH1, welches $\mathrm{zu}$ einer verminderten Hydroxylierung eines Lysinsrests und einer gestörten Kollagenvernetzung führt, kommt es $\mathrm{zu}$ einer reduzierten Kollagensekretion sowie zu einer Differenzierungsstörung der Osteoblasten. Die wenigen bekannten Patienten haben eine mittelschwere bis schwere OI [20].

\section{CREB3L1 - OI-Typ XVI}

CREB3L1 (cyclic AMP-responsive element-binding protein 3-like protein 1) kodiert einen Transkriptionsfaktor (CR3L1, früher OASIS). Im Zusammenhang mit ER-Stress wird ein Fragment von CR3L1 durch eine Metalloprotease (S1P, S2P; siehe OI-Typ XIX) freigesetzt. Dieses induziert im Nukleolus die Synthese von Proteinen der „unfolded protein response“ (UPR). Dies ist unter anderem spezifisch für eine „UPRelement-like-Sequenz" in einer osteoblastenspezifischen COL1A1-Promotorregion, nicht aber in der entsprechenden hautspezifischen COL1A1-Promotoregi- 
on. Deshalb kommt es bei Patienten zu einer reduzierten Kollagenproduktion in Knochen-, aber nicht Hautzellen, teilweise begleitet von veränderter Zusammensetzung und Hypermineralisierung der Knochenmatrix. Biallelisch Betroffene zeigen meist einen mittelschweren bis schweren Verlauf, häufig mit bereits pränatal sichtbaren Frakturen und Verkürzungen der langen Röhrenknochen [21]. Es gibt in den bekannten Familien aber auch heterozygote, leichter Betroffene, mit postpartalem Beginn und Gehfähigkeit. Manche der heterozygot Betroffenen sind klinisch unauffällig.

\section{SEC24D}

SEC24D kodiert eine Proteinkomponente des COPII-abhängigen ER-zu-GolgiTransports. Eine Mutation in diesem Gen führt molekular zu einer Retention des Prokollagens im ER und klinisch zu einer gestörten Ossifikation des Schädelknochens mit kraniofazialen Fehlbildungen und Frakturneigung zum Teil mit pränatalem Beginn. Diese Symptomkonstellation wird als Cole-Carpenter-Syndrom Typ 2 bezeichnet [22]. Andere Fälle wurden klinisch als OI diagnostiziert, u.a. als klassische OI mit graublauen Skleren, Schaltknochen und verkürzten bzw. verbogenen Röhrenknochen. Diese Patienten können laufen und fast normal am Leben teilhaben.

\section{SPARC - OI-Typ XVII}

Zwei Punkt-Missense-Mutationen wurden bislang im SPARC-Gen beschrieben. Diese führen zu einem Austausch von Aminosäuren im SPARC-Protein (secreted protein, acidic and rich in cysteine, alternativ auch als Osteonectin bezeichnet), die essenziell für die Bindung zwischen SPARC und Kollagen sind. SPARC kann intrazellulär als molekulares Chaperon dienen und extrazelluläre Zell-Matrix-Interaktionen vermitteln. In Patientenzellen kommt es entsprechend zu einer geringen Übermodifikation und einer leicht verzögerten Sekretion des Kollagens. Aber auch außerhalb der Zelle übt SPARC als ein vorrangig extrazelluläres Protein eine Funktion aus, indem es an das Kollagen und an Hydroxylapatitkristalle bindet und die Mineralisierung der extrazellulären Matrix för- dert. Damit erfüllt SPARC eine multivalente Rolle in der Aufrechterhaltung der Knochenmasse und -qualität. Die beiden erstbeschriebenen Patienten mit SPARCMutationen sind mittelschwer betroffene Patienten [23].

\section{BMP1 - OI-Typ XIII}

Nach der Sekretion des Kollagens in den Extrazellularraum ist die proteolytische Abspaltung des C-terminalen Propeptids notwendig, um eine Kollagenassemblierung und die Fibrillenbildung zu ermöglichen. Mutationen in COL1A1 oder COL1A2, die an einer entsprechenden Spaltungsstelle liegen, führen zu einer leichten OI-Form, gekennzeichnet durch erhöhte Knochenmasse. Mutationen im $B M P 1-G e n$, das eine für die Spaltung verantwortliche Protease (bone morphogenetic protein 1) kodiert, resultieren in einer OI mit einem sehr variablen, von leicht bis schwer betroffenen Phänotyp. In Patientenzellen ist die Prozessierung des Prokollagens und die Fähigkeit, reife Kollagenfibrillen zu generieren, eingeschränkt. Dies ist mit einer gesteigerten Mineralisierung der Kollagenmatrix und erhöhter Knochenmasse assoziiert [24].

\section{Osteogenesis imperfecta und Osteoblastendifferenzierungs- störungen}

Gene, die an der Differenzierung der knochenproduzierenden Osteoblasten beteiligt sind, wurden in den vergangenen Jahren mit OI assoziiert, jedoch wurde in den meisten Fällen der Pathomechanismus noch nicht vollständig aufgeklärt.

\section{SP7 - OI-Typ XII}

Mutationen im Gen SP7, das den osteoblastenspezifischen Transkriptionsfaktor Sp7 (oder Osterix) kodiert und die Differenzierung von Prä-Osteoblasten zu Osteoblasten sowie Osteozyten einleitet, führen zu einer eher leichten Verlaufsform mit wiederholten Frakturen und sind zum Teil mit Schwerhörigkeit assoziiert. Diese Patienten weisen eine erhöhte Knochenporosität auf, die auf einen erhöhten trabekulären Knochenumbau zurückgeführt werden könnte [25].

\section{WNT1 - OI-Typ XV}

Mutationen im Gen des sekretierten Glykoproteins WNT1 (wingless-type MMTV integration site family 1), das den WNT-Signalweg induziert, führen $\mathrm{zu}$ einer OI mit verschiedenen klinischen Schweregraden. Nach Bindung von WNT1 an den dualen Rezeptorkomplex aus LRP5/6 und Frizzled wird der sekundäre Botenstoff $\beta$-Catenin stabilisiert und kann in den Nukleus translozieren, um die Expression von Genen zu regulieren, die an der Osteoblastendifferenzierung und -aktivität beteiligt sind. Durch Mutationen im WNT1-Gen kommt es zur veränderten Signalweiterleitung und eingeschränkter Expression von osteoblastenspezifischen Genen, welche die Knochenzellhomöostase regulieren. Bei histopathologisch unauffälliger Knochenmineralisation zeigt sich klinisch/laborchemisch ein Missverhältnis von Knochenauf- und -abbau [26]. In Mausmodellen konnte belegt werden, dass das Fehlen eines funktionellen WNT1 in Osteoblasten für die Manifestierung von OI verantwortlich ist. Da WNT1 ebenfalls im Gehirn exprimiert ist, sind Patienten oft auch von Entwicklungsstörungen des zentralen Nervensystems betroffen. Zusätzlich $\mathrm{zu}$ den unterschiedlich stark ausgeprägten kognitiven Einschränkungen haben die Patienten einen mittelschweren bis schweren Knochenphänotyp. Interessanterweise führen Mutationen im WNT1Rezeptor LRP5 (low density lipoprotein receptor-related protein 5) zu dem Osteoporose-Pseudogliom-Syndrom mit überlappenden Knochenmerkmalen einer OI.

\section{TENT5A - OI-Typ XVIII}

Kürzlich wurden Mutationen im TENT5A-Gen (früher auch als FAM46A bezeichnet), welches die terminale $\mathrm{Nu}$ kleotidyltransferase 5A kodiert, als OIauslösend in drei Patienten beschrieben und eine bis dato unbekannte Funktion dieses Enzyms im mineralisierten Gewebe gezeigt. Die bisher nur in einer Publikation beschriebenen Patienten und Patientinnen sind mittelschwer bis schwer betroffen [27]. Die Expression von TENT5A in Osteoblasten legt eine Rolle in der Knochenhomöosta- 
se nahe und wird durch die Analyse eines neuen Mausmodells für skelettale Dysplasien unterstützt. Die Mäuse tragen eine TENT5A-Mutation und entwickeln einen OI-ähnlichen Phänotyp, der Pathomechanismus dahinter wurde allerdings noch nicht aufgeklärt. Untersuchungen im Krallenfrosch (Xenopus) zeigen aber, dass das TENT5A-Protein den für Knochenbildung und -homöostase essenziellen BMP-Signalweg durch eine Stabilisierung des Effektor-SMAD aktiviert.

\section{Weitere genetische Ursachen}

\section{IFITM 5 - OI-Typ V}

IFITM5 ist ein Gen für das gleichnamige Protein „interferon-induced transmembrane protein-5“, früher BRIL (bonerestricted interferon-induced transmembrane protein-like protein). Heterozygote Mutationen in IFITM5 resultieren - neben den klassischen Kollagenmutationen - in der einzigen weiteren autosomal dominanten OI-Form. Die Funktion von IFITM5, mehr noch der Pathomechanismus der von den bekannten Mutationen ausgelösten Veränderungen, ist bis heute nicht vollständig geklärt. IFITM5 scheint in der Osteoblastendifferenzierung und der Knochenmineralisation eine Rolle zu spielen. In Osteoblastenkulturen mit vergleichbaren Mutationen konnte eine Osteoblastendifferenzierungsverzögerung, eine verminderte Menge an Kollagen und eine erhöhte Mineralisation gezeigt werden. Die erste bekannte und häufigste Mutation beim Menschen (5'-nichttranslatierte Region, neues Startcodon), welche zu einer "Gain-of-function-Mutation“ führt, ist verantwortlich für die typischen OITyp-V-Symptome. Dazu zählen die hyperplastische Kallusbildung im Rahmen von Frakturen und die übermäßige Ossifikation der Membrana interossea an den Unterarmen [28]. Zusätzlich kommen Radiusköpfchenluxationen vor. Daneben gibt es Mutationen in der kodierenden Region von IFITM5, welche $\mathrm{zu}$ einer reduzierten Mineralisation führen, ähnlich wie bei etablierten Mausmodellen. Weiterhin gibt es eine bisher unklare Interaktion zwischen IFITM5 und PEDF, welche sich bei Patienten mit einer nicht klassischen IFITM5-Mutation zeigten, die sich klinisch ähnlich der OI-Typ VI präsentierten.

\section{SERPINF1 - OI-Typ VI}

Eine Sonderstellung unter den rezessiven Formen der OI nimmt das Gen SERPINF1 ein, da es nicht auf die Kollagenbildung in den Osteoblasten einwirkt, sondern den Knochenabbau steigert. SERPINF1 kodiert für das Protein „pigment epithelium-derived factor“ (PEDF). Ein Funktionsverlust von SERPINF1 führt zu einer vermehrten Differenzierung und Aktivierung von Osteoklasten, vermittelt über das RANK/OsteoprotegerinSystem. Es liegt also ein vermehrter Abbau vor [29]. Diese Patienten haben in der Regel keine perinatalen Frakturen, sondern erste Frakturen treten erst mit 4-18 Monaten auf. Die Frakturhäufigkeit und die Schwere der Erkrankung sind progredient. Dabei sind die Skleren und die Zähne meistens unauffällig [30]. Aus der Überaktivierung der Osteoklasten ergibt sich ein anderer therapeutischer Ansatz, der eine gezieltere Behandlung auf Grundlage der Pathophysiologie ermöglicht (s. Therapeutische Perspektiven Denosumab).

\section{PLS3}

PLS3 ist ein $\mathrm{X}$-chromosomales Gen, welches für ein F-Aktin bindendes $\mathrm{Zy}$ toskelettprotein kodiert (Plastin-3). Eine Mutation führt $\mathrm{zu}$ einer frühmanifestierenden Osteoporose, in schweren Verlaufsformen kann es auch zu einem OI-ähnlichen Phänotyp führen. Durch den X-chromosomalen Erbgang sind in der Regel männliche Patienten schwerer betroffen. Plastin-3 ist an der Bildung von F-Aktin-Bündeln beteiligt und spielt im intrazellulären Signalweg „NF-kB repressing factor“ (NKRF) in Osteoklasten eine Rolle. Es ist ubiquitär, insbesondere in allen Knochenzellreihen, exprimiert. Plastin-3 scheint eine Rolle in der Knochenbildung und Knochenmineralisation und im Knochenabbau zu spielen. Pathomechanistisch wird eine verminderte Mechanotransduktion aufgrund der gestörten Interaktion der extrazellulären Matrix mit dem Zytoskelett der Knochenzellen, insbesondere der Osteozyten, angenommen. Außerdem sind die Resorptionsaktivität, die Adhäsion und Migration von Osteoklasten unmittelbar von der Bildung großer Aktinfilamente abhängig. Mutationen in PLS3 führen $\mathrm{zu}$ einer reduzierten trabekulären Dicke des Knochens bei normaler Expression und Modifikation von Kollagen sowie zu einem normalen Muster der Knochenlamellenstruktur. Diese Veränderung spricht, wie andere klinische und laborchemische Befunde, für eine Überaktivität von Osteoklasten als teilweise Grundlage für den Knochenphänotyp. Zusätzlich haben die Patienten eine normale, zum Teil auch reduzierte Mineralisation, obwohl in den meisten anderen OI-Formen eine eher kompensatorische Hypermineralisation vorliegt [31].

\section{Therapeutische Perspektive}

\section{Denosumab}

Die Identifizierung von SERPINF1 und die sich daraus ergebende Pathophysiologie mit einer vermehrten Osteoklastenaktivierung eröffnete neue therapeutische Möglichkeiten. Der monoklonale Antikörper Denosumab, damals nur für die Behandlung der postmenopausalen Osteoporose zugelassen, greift in den Regulierungsprozess der OPG/RANKLKaskade ein, in dem die Ursache für den gesteigerten Knochenabbau liegt. Erste Fallserien zeigten, dass Denosumab bei Patienten mit OI-Typ VI wesentlich zielgerichteter wirkt und eine größere $\mathrm{Zu}$ nahme an Knochenmasse bewirkt als eine Therapie mit Bisphosphonaten [32]. Da es sich beim Einsatz von Denosumab um eine wesentlich direktere antiresorptive Wirkung handelt, treten auch stärkere Schwankungen des Serum-Kalziumspiegels mit einer Hypokalzämie in den ersten 4 Wochen nach Gabe und eine ReboundHyperkalzämie nach ca. 3-4 Monaten auf. Dies muss beachtet werden, da diese Schwankungen sehr viel ausgeprägter sind als unter Bisphosphonaten. Dafür ist der Antikörper nach einigen Monaten komplett aus dem Organismus abgebaut und bietet - im Gegensatz zu Bis- 
phosphonaten - vermutlich ein geringeres Risiko für langfristige Nebenwirkungen [33]. Derzeit wird eine internationale Zulassungsstudie zum Einsatz von Denosumab bei Kindern und Jugendlichen mit OI (NCT02352753) durchgeführt, deren Ergebnisse vermutlich 2021 vorliegen werden.

\section{Anti-Sklerostin}

Sowohl Bisphosphonate als auch Denosumab verwirklichen einen antiresorptiven Ansatz, der dazu führt, dass mehrvon dem von den Osteoblasten produzierten Knochen erhalten bleibt. Eine Stimulation des Knochenaufbaus gibt es derzeit für das Kindes- und Jugendalter noch nicht. Im Erwachsenenbereich gibt es Untersuchungen zur Wirksamkeit von Parathormon als osteoanaboles Medikament, das bei Erwachsenen mit OI eine $\mathrm{Zu}$ nahme der Knochensubstanz erreichen konnte [34]. Aufgrund von Malignomen im Tierversuch beim wachsenden Skelettsystem wurde dieses Medikament jedoch nie bei Kindern vor Verschluss der Wachstumsfugen angewendet [35].

Eine andere osteoanabole Substanz könnte in Zukunft mit dem Wirkstoff Anti-Sklerostin verfügbar werden. Sklerostin greift hemmend in die Differenzierung von Osteoblasten ein. Deshalb könnte über eine Blockierung von Sklerostin die Zahl und Aktivität von Osteoblasten gesteigert werden. Eine erste Studie mit kurzer Laufzeit bei Erwachsenen mit OI hat in Bezug auf die Laborparameter entsprechende Ergebnisse geliefert [36]. Ob die Veränderungen der Knochenstoffwechselmarker mit einer Senkung der Frakturrate oder der Knochenschmerzen korrelieren, ist noch nicht abschließend untersucht. Die Untersuchung der Wirksamkeit und Sicherheit eines solchen Wirkstoffs bei Kindern mit OI befindet sich derzeit in Vorbereitung (NCT01417091). Allerdings sollte vorher der onkogene Effekt, der sich beim Parathormon zeigte, ausgeschlossen werden und es muss geprüft werden, wie die Sklerostinspiegel bei OI-Patienten sind. Aus pathophysiologischer Sicht wäre es sinnvoll, wenn der Körper bei einem instabilen Ske- lettsystem die Sklerostinspiegel selber herunterreguliert.

\section{Therapeutische Perspektive Stammzellen}

Egal ob antiresorptive Therapien oder osteoanabole Ansätze für die medikamentöse Behandlung genutzt werden, das Ergebnis ist immer eine Zunahme von Knochensubstanz, die allerdings bei schwer betroffenen Patienten in ihrer Qualität weiter reduziert ist. Da aufgrund der Vielzahlder Mutationen eine gezielte Gentherapie in weiter Ferne liegt, gibt es derzeit erste Ansätze einer Stammzelltherapie. Durch eine möglichst frühe Gabe embryonaler, mesenchymaler Stammzellen, die als nicht immunogen gelten, könnten im Körper der Betroffenen Osteoblasten entstehen, die ein von der Qualität her normales Kollagen produzieren. Erste Fallberichte scheinen einen Nutzen zumindest über einige Monate zu belegen [37]. Derzeit wird im Rahmen eines EU-weiten Forschungsprojektes in einigen Zentren die Sicherheit und Wirksamkeit einer solchen Therapie untersucht (NCT03706482).

Therapeutische Perspektive TGF- $\beta$ Antikörper

Die Rolle von TGF- $\beta$ bei Störungen der extrazellulären Matrix wird seit vielen Jahren untersucht. Grafe et al. beschreiben die Rolle von TGF- $\beta$ in OI-Mausmodellen als einen primären Pathomechanismus bei dominanten und rezessiven OI-Formen. Aktuell läuft eine multizentrische, klinische Studie zur Sicherheit von TGF- $\beta$-Antikörper bei Erwachsenen mit Osteogenesis imperfecta (NCT03064074).

\section{Fazit für die Praxis}

Die Osteogenesis imperfecta ist eine Multisystemerkrankung, die durch eine erhöhte Frakturgefahr sowie durch muskulo-ligamentäre Probleme gekennzeichnet ist. Die Therapie besteht aus einem interdisziplinären Ansatz aus orthopädischen, medikamentösen und physiotherapeutischen Maßnahmen. Dabei ist die Therapie derzeit unabhän- gig von der molekulargenetischen Ursache. In den letzten Jahren wurden verschiedene Gene entdeckt, was zu einem neuen pathophysiologischen Verständnis der seltenen Ol-Formen geführt hat. Hierbei gibt es Formen, bei denen eine Störung der posttranslationalen Modifikation, der Ausschleusung von Kollagen aus der Zelle oder eine Überaktivierung der Osteoklasten vorliegt. Aus diesem neuen Verständnis werden derzeit neue therapeutische Ansätze (Denosumab, osteoanabole Therapie) entwickelt und auch erste Untersuchungen zum Einsatz von Stammzellen bei OI unternommen.

\section{Korrespondenzadresse}

\section{Oliver Semler}

Medizinische Fakultät und Uniklinik Köln, Klinik und Poliklinik für Kinder- und Jugendmedizin, Universität zu Köln

Kerpenerstraße 62, 50931 Köln, Deutschland joerg.semler@uk-koeln.de

Förderung. Teile dieser Arbeit wurden unterstützt durch die DFG-Förderung FOR 2722.

\section{Einhaltung ethischer Richtlinien}

Interessenkonflikt. M. Rehberg, J. Etich, L. Leßmeier H. Sill, C. Netzer und O. Semler geben an, dass kein Interessenkonflikt besteht.

Für diesen Beitrag wurden von den Autoren keine Studien an Menschen oder Tieren durchgeführt. Für die aufgeführten Studien gelten die jeweils dort angegebenen ethischen Richtlinien.

Open Access. Dieser Artikel wird unter der Creative Commons Namensnennung 4.0 International Lizenz veröffentlicht, welche die Nutzung, Vervielfältigung, Bearbeitung, Verbreitung und Wiedergabe in jeglichem Medium und Format erlaubt, sofern Sie den/die ursprünglichen Autor(en) und die Quelle ordnungsgemäß nennen, einen Link zur Creative Commons Lizenz beifügen und angeben, ob Änderungen vorgenommen wurden.

Die in diesem Artikel enthaltenen Bilder und sonstiges Drittmaterial unterliegen ebenfalls der genannten Creative Commons Lizenz, sofern sich aus der Abbildungslegende nichts anderes ergibt. Sofern das betreffende Material nicht unter der genannten Creative Commons Lizenz steht und die betreffende Handlung nicht nach gesetzlichen Vorschriften erlaubt ist, ist für die oben aufgeführten Weiterverwendungen des Materials die Einwilligung des jeweiligen Rechteinhabers einzuholen.

Weitere Details zur Lizenz entnehmen Sie bitte der Lizenzinformation auf http://creativecommons.org/ licenses/by/4.0/deed.de. 


\section{Literatur}

1. Marini JC, Forlino A, Bachinger HP, Bishop NJ, Byers PH, Paepe A et al (2017) Osteogenesis imperfecta. Nat Rev Dis Primers 3:17052

2. Hoyer-Kuhn H, Bartz-Seel J, Blickheuser R, v. Deimling U, Stücker R, Wirth T et al (2016) Diagnostik und Therapie der Osteogenesis imperfecta. Monatsschr Kinderheilkd 165(4):333-346

3. Devaraju D, Devi BY, Vasudevan V, Manjunath V (2014) Dentinogenesis imperfecta type I: a case report with literature review on nomenclature system. JOral Maxillofac Pathol 18(1):131-134

4. Krohn K, Schwartz EN, Chung YS, Lewiecki EM (2019) Dual-energy X-ray absorptiometry monitoring with trabecular bone score: the 2019 ISCD official positions. J Clin Densitom 22(4):501-505. https://doi.org/10.1016/j.jocd.2019.07.006

5. Glorieux FH, Bishop NJ, Plotkin H, Chabot G, Lanoue G, Travers R (1998) Cyclic administration of pamidronate in children with severe osteogenesis imperfecta. NEngl J Med 339(14):947-952

6. Dwan K, Phillipi CA, Steiner RD, Basel D (2016) Bisphosphonate therapy for osteogenesis imperfecta. Cochrane Database Syst Rev 10:CD5088

7. Land C, Rauch F, Montpetit K, Ruck-Gibis J, Glorieux $\mathrm{FH}$ (2006) Effect of intravenous pamidronate therapy on functional abilities and level of ambulation in children with osteogenesis imperfecta. JPediatr 148(4):456-460

8. Maines E, Monti E, Doro F, Morandi G, Cavarzere P, Antoniazzi F (2012) Children and adolescents treated with neridronate for osteogenesis imperfecta show no evidence of any osteonecrosis of the jaw. JBone Miner Metab 30(4):434-438

9. Wirth T (2012) Osteogenesis imperfecta. Orthopade 41(9):773-782 (quiz 83-4)

10. Semler O, Fricke O, Vezyroglou K, StarkC, Stabrey A, Schoenau E (2008) Results of a prospective pilot trial on mobility after whole body vibration in children and adolescents with osteogenesis imperfecta. Clin Rehabil 22(5):387-394

11. Mueller B, Engelbert R, Baratta-Ziska F, Bartels B, Blanc N, Brizola E et al (2018) Consensus statement on physical rehabilitation in children and adolescents with osteogenesis imperfecta. Orphanet JRare Dis 13(1):158

12. Veilleux LN, Pouliot-Laforte A, Lemay $M$, Cheung MS, Glorieux FH, Rauch F (2015) The functional muscle-bone unit in patients with osteogenesis imperfecta type I. Bone 79:52-57

13. Ben Amor IM, Roughley P, Glorieux FH, Rauch F (2013) Skeletal clinical characteristics of osteogenesis imperfecta caused by haploinsufficiency mutations in COL1A1. J Bone Miner Res 28(9):2001-2007

14. Kang $\mathrm{H}$, Aryal ACS, Marini JC (2017) Osteogenesis imperfecta: new genes reveal novel mechanisms in bone dysplasia. Transl Res 181:27-48

15. Besio R, Chow CW, Tonelli F, Marini JC, Forlino A (2019) Bone biology: insights from osteogenesis imperfecta and related rare fragility syndromes. FEBS J 286(15):3033-3056

16. Ward LM, Rauch F, Travers R, Chabot G, Azouz EM, Lalic L et al (2002) Osteogenesis imperfecta type VII: an autosomal recessive form of brittle bone disease. Bone 31(1):12-18

17. Duran I, Martin JH, Weis MA, Krejci P, Konik P, Li B et al (2017) A chaperone complex formed by HSP47, FKBP65, and BiP modulates telopeptide lysyl hydroxylation of type I procollagen. J Bone Miner Res 32(6):1309-1319

18. Christiansen HE, Schwarze U, Pyott SM, AISwaid A, Al Balwi M, Alrasheed S et al (2010) Homozygosity for a missense mutation in SERPINH1, which encodes the collagen chaperone protein HSP47, results in severe recessive osteogenesis imperfecta. Am J Hum Genet 86(3):389-398

19. Duran I, Nevarez L, Sarukhanov A, Wu S, Lee K, Krejci Petal (2015) HSP47 and FKBP65 cooperate in the synthesis of type I procollagen. Hum Mol Genet 24(7):1918-1928

20. Lindert U, Cabral WA, Ausavarat S, Tongkobpetch S, Ludin K, Barnes AM et al (2016) MBTPS2 mutations cause defective regulated intramembrane proteolysis in X-linked osteogenesis imperfecta. Nat Commun 7:11920

21. Symoens $S$, Malfait F, D'Hondt $S$, Callewaert B, Dheedene A, Steyaert W et al (2013) Deficiency for the ER-stress transducer OASIS causes severe recessive osteogenesis imperfecta in humans. Orphanet J Rare Dis 8(1):154

22. Garbes L, Kim K, Riess A, Hoyer-Kuhn H, Beleggia F, Bevot A et al (2015) Mutations in SEC24D, encoding a component of the COPII machinery, cause a syndromic form of osteogenesis imperfecta. Am J Hum Genet 96(3):432-439

23. Mendoza-Londono R, Fahiminiya S, Majewski J, Care4Rare Canada Consortium, TetreaultM, Nadaf J et al (2015) Recessive osteogenesis imperfecta caused by missense mutations in SPARC. Am JHum Genet 96(6):979-985

24. Martinez-Glez V, Valencia M, Caparros-Martin JA, Aglan M, Temtamy S, Tenorio J et al (2012) Identification of a mutation causing deficient BMP1/mTLD proteolytic activity in autosomal recessive osteogenesis imperfecta. Hum Mutat 33(2):343-350

25. Lapunzina P, Aglan M, Temtamy S, CaparrosMartin JA, Valencia M, Leton R et al (2010) Identification of a frameshift mutation in osterix in a patient with recessive osteogenesis imperfecta. Am JHum Genet 87(1):110-114

26. Pyott SM, Tran TT, Leistritz DF, Pepin MG, Mendelsohn NJ, Temme RT et al (2013) WNT1 mutations in families affected by moderately severe and progressive recessive osteogenesis imperfecta. Am J Hum Genet 92(4):590-597

27. Doyard M, Bacrot S, Huber C, Di Rocco M, GoldenbergA, Aglan MSetal (2018) FAM46A mutations are responsible for autosomal recessive osteogenesis imperfecta. J Med Genet 55(4):278-284

28. Semler O, Garbes L, Keupp K, Swan D, Zimmermann K, Becker J et al (2012) A mutation in the $5^{\prime}$-UTR of IFITM5 creates an in-frame start codon and causes autosomal-dominant osteogenesis imperfecta type $V$ with hyperplastic callus. Am J Hum Genet 91(2):349-357

29. Becker J, Semler O, Gilissen C, Li Y, Bolz HJ, Giunta $C$ et al (2011) Exome sequencing identifies truncating mutations in human SERPINF1 in autosomal-recessive osteogenesis imperfecta. Am JHum Genet 88(3):362-371

30. Glorieux FH, Ward LM, Rauch F, Lalic L, Roughley PJ, Travers R (2002) Osteogenesis imperfecta type VI: a form of brittle bone disease with a mineralization defect.J Bone Miner Res 17(1):30-38

31. van Dijk FS, Zillikens MC, Micha D, Riessland $M$, Marcelis CL, de Die-Smulders CE et al (2013) PLS3 mutations in X-linked osteoporosis with fractures. NEngl J Med 369(16):1529-1536

32. Hoyer-Kuhn H, Netzer C, Koerber F, Schoenau E, Semler $O$ (2014) Two years' experience with denosumab for children with osteogenesis imperfecta type VI. Orphanet J Rare Dis 9(1):145

33. Hoyer-Kuhn H, Franklin J, Allo G, Kron M, Netzer C, Eysel P et al (2016) Safety and efficacy of denosumab in children with osteogenesis imperfect-a first prospective trial. J Musculoskelet Neuronal Interact 16(1):24-32

34. Orwoll ES, Shapiro J, Veith S, Wang Y, Lapidus J, Vanek C et al (2014) Evaluation of teriparatide treatment in adults with osteogenesis imperfecta. JClin Invest 124(2):491-498

35. Kuijpers G, Schneider B, Stadel B, Colman E (2002) Recombinant human parathyroid hormone. Preclinical data on rat osteosarcoma were not dismissed. BMJ 324(7347):1218 (author reply)

36. Glorieux FH, Devogelaer JP, Durigova M, Goemaere S, Hemsley S, Jakob F et al (2017) BPS804 anti-sclerostin antibody in adults with moderate osteogenesis Imperfecta: results of a randomized phase 2a trial. J Bone Miner Res 32(7):1496-1504

37. Gotherstrom C, Westgren M, Shaw SW, Astrom E, Biswas A, Byers PH et al (2014) Pre- and postnatal transplantation of fetal mesenchymal stem cells in osteogenesis imperfecta: a two-center experience. Stem Cells Transl Med 3(2):255-264 
Hier steht eine Anzeige.

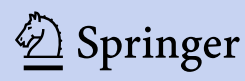

